\title{
PREVENTING OXIDATION OF CANOLA AND SUNFLOWER OILS BY ADDITION OF POMEGRANATE SEED OIL
}

\author{
N. SiRaj ${ }^{\mathrm{a} *}$, M.A. ShabBIR ${ }^{\mathrm{a}}$, M.R. KHAN ${ }^{\mathrm{a}}$ and K.U. ReHMAN ${ }^{\mathrm{b}}$

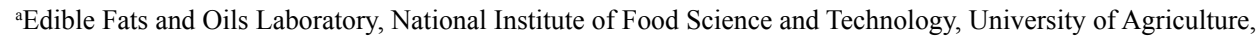 \\ Faisalabad, 38000. Pakistan \\ ${ }^{b}$ Department of Biochemistry, Faculty of Sciences, University of Agriculture, Faisalabad, 38000 Pakistan
}

(Received: 14 February 2018; accepted: 4 June 2018)

\begin{abstract}
The study was designed to explore the antioxidative effect of pomegranate seed oil (PSO) at different concentrations ( 5 and 7\%) against oxidation of plant-based oils (canola oil and sunflower oil) during storage (60 days) as compared to artificial antioxidant butylated hydroxyanisole (BHA, $200 \mathrm{ppm}$ ). Rancimat and Schaal oven analysis were employed for the assessment of potential consequences of PSO against oxidation in plant based oils. The variation in total phenolic contents (TPC), antioxidant activity, peroxide value (POV), and tocopherol contents during storage were evaluated by Schaal oven test at $62{ }^{\circ} \mathrm{C}$. The substantially $(\mathrm{P} \leq 0.05)$ higher induction period (IP) values were observed for PSO blended oil samples as compared to blank oil samples. The addition of PSO in plant-based oils improved the oxidative stability by enhancing the antioxidant potential and TPC, decreasing POV, and slowing down the degradation of tocopherol contents during storage. The findings of the present study suggest that PSO might be used as an alternative potential antioxidant to synthetic antioxidants.

Keywords: canola oil, oxidative stability, pomegranate seed oil, Rancimat analysis, Schaal oven test,
\end{abstract} sunflower oil

Lipid autoxidation is a major quality deterioration factor that effects the nutritional and sensorial quality of oils/fats (DA SILVA \& JORGE, 2014). To prevent the oxidation process, various artificial antioxidants, i.e. butylated hydroxyanisole (BHA), tert-butyl hydroxylquinone (TBHQ), and butylated hydroxytoluene (BHT), were added in edible fats/ oils due to their low cost and high oxidative stability, but utilization of these antioxidants elevated health issues as proved by various studies (TAGHVAEI \& JAFARI, 2015). Addition of natural antioxidant can be beneficial for retarding oxidation process by capturing free radicals and decomposing peroxides (Sun-Waterhouse et al., 2011). In the last two decades, application of antioxidants from natural sources, i.e. olive oil mill wastewater, rosemary extract, etc., were employed in edible oils with potential protective effects (JenNINGS \& Акон 2009; AbD-Elghany et al., 2010).

A whole pomegranate fruit is composed of $50-64 \%$ edible portion, which is further divided into $35-40 \%$ juicy aril portion and $10-15 \%$ seed portion that is considered as waste after juice extraction (VIUDA-MARTOS et al., 2010). This seed portion possesses unique fatty acid octadecatrienoic omega-5 fatty acid (punicic acid), 3,3'-di-O-methylellagic acid, sterols, and tocopherols (Lansky \& Newman, 2007; Sreekumar et al., 2014). Pomegranate byproducts possess potential glycemic, antioxidant, and antimicrobial activity as reported by various studies (TeHRANIFAR et al., 2011). Though pomegranate bagasse contains bioactive components, its application in food industry is scarce.

* To whom correspondence should be addressed. Phone: +92-300-8431773; e-mail: nailaateeq02@gmail.com 
The Rancimat analysis is a widely used method for evaluating the antioxidative activities of antioxidants, and with less labor and time, it is a better alternative to active oxygen method (YANG et al., 2016). It measures the changes in conductivities generated by lower molecular volatile organic acids produced during oxidation process in oils/fats under high temperature (Cordeiro et al., 2013). The time duration that passes till secondary oxidation occurs is known as IP, and it measures the resistance of the oil to oxidation.

The aim of the study was to compare the antioxidative effects of PSO and artificial antioxidant on the storage stability of plant-based edible oils analysed with the Rancimat and Schaal oven test.

\section{Materials and methods}

\subsection{Procurement of raw materials}

Canola oil and sunflower oil without any artificial antioxidants were procured from Hao Koufu Co., Ltd., Shanxi, China. Tocopherol standards, butylated hydroxyanisole (BHA), and all other reagents of analytical grades were purchased from Sigma-Aldrich (St Louis, MO, USA) and other standard companies.

\subsection{Extraction of pomegranate seed oil}

Pomegranate seeds were separated from peels and other adhering materials by using a juice extractor (Ju 2000 Vitae, Moulinex, Barcelona, Spain). To inactivate the lipolytic enzyme's activity, seeds were washed and then dried in hot air oven at $140{ }^{\circ} \mathrm{C}$ for $20 \mathrm{~min}$. The seeds were grinded and passed through 30-40 mesh screens to obtain seed powder (RUGE et al., 2012). For the extraction of oil, approx. $20 \mathrm{~g}$ seed powder was homogenized with $100 \mathrm{ml}$ of chloroform and methanol solution $(1 / 1, v / v)$ in a screw capped glass bottle. The sample bottle was kept at $60^{\circ} \mathrm{C}$ for $20 \mathrm{~min}$, afterwards $100 \mathrm{ml}$ chloroform was added again and homogenized. After $3 \mathrm{~min}$ of homogenization, the mixture was filtered. The filtrate was mixed with $70 \mathrm{ml}$ of $1 \mathrm{M} \mathrm{KCl}$ solution and kept at $4{ }^{\circ} \mathrm{C}$ overnight for phase separation. The organic phase was collected and dried at $40{ }^{\circ} \mathrm{C}$ in a rotary evaporator, then dissolved in $5 \mathrm{ml} \mathrm{n}$-hexane/ isopropanol solution $(4 / 1, \mathrm{v} / \mathrm{v})$. The extracted oil samples were stored at $-18{ }^{\circ} \mathrm{C}$ in amber glass jars for further analysis.

\subsection{Rancimat analysis}

PSO was directly incorporated in canola and sunflower oils at 5 and 7\% level and stirred for $10 \mathrm{~min}$ at ambient temperature. The Rancimat 892 instrument (Metrohm, Herisau, Switzerland) was used for the analysis. Both oils with artificial antioxidant (BHA) addition (200 ppm) were considered as positive controls and oil without any incorporation was considered as negative control. Each oil sample (approx. $5 \mathrm{~g}$ ) was added to all reaction vessels and run under standard conditions (temp. $120^{\circ} \mathrm{C}$ and airflow rate $20 \mathrm{l} \mathrm{h}^{-1}$ ). The results were calculated from the inflection point of the curve automatically by the software provided with the instrument and expressed in IP (YANG et al., 2016). 


\subsection{Schaal oven storage stability test}

From each oil sample approx. $50 \pm 0.01 \mathrm{~g}$ was weighed into tightly closed amber glass jars with no headspace, kept in a hot air oven at $62{ }^{\circ} \mathrm{C}$ for 60 days. From each jar a sample was withdrawn at every 10 days for evaluating antioxidant activity and oxidative stability index, while the tocopherol contents were examined at 0 and 60 days (AnTOLOvich et al., 2002).

\subsection{Antioxidant activity}

1.5.1. DPPH radical scavenging analysis. The Trolox standard/oil sample $(0.1 \mathrm{ml})$ was added to $3.9 \mathrm{ml} \mathrm{DPPH}$ stock solution $\left(6 \times 10^{-5} \mathrm{M}\right)$ and left at room temperature for $2 \mathrm{~h}$ in dark. The absorbance of the mixture was measured by spectrophotometer (UV Mini 1240, Shimadzu, Kyoto, Japan) at $515 \mathrm{~nm}$ wavelength. Methanol was used as blank. Results were presented as $\mathrm{mg} \mathrm{TE} / \mathrm{ml}$ oil sample (Sur et al., 2014).

1.5.2. ABTS radical scavenging analysis. The ABTS stock solution $(7 \mathrm{mM})$ was reacted with an equal volume of potassium persulphate $(2.45 \mathrm{mM})$ for preparing ABTS radical cation solution, and was left at room temperature for $12-16$ hours in a dark place. One millilitre of ABTS radical cation solution was mixed with $60 \mathrm{ml}$ methanol solution, getting an absorbance of $0.70 \pm 0.02$ at $734 \mathrm{~nm}$ wavelength. The Trolox standard/sample aliquot $(2.5 \mu \mathrm{l})$ was reacted with $1 \mathrm{ml}$ ABTS radical cation solution for $7 \mathrm{~min}$, then readings were obtained from the spectrophotometer. Results were presented as $\mathrm{mg}$ TE/ml oil sample (SuI et al., 2014).

1.5.3. Total phenolic contents (TPC). The Folin-Ciocalteu reagent $(0.5 \mathrm{ml})$ and $2 \mathrm{ml}$ methanol were added to the oil sample aliquots $(100 \mathrm{mg})$ and shaken well. Afterwards, $15 \%$ $\mathrm{Na}_{2} \mathrm{CO}_{3}(1.5 \mathrm{ml})$ was mixed with the mixture for $30 \mathrm{~s}$, then it was filled up to $7 \mathrm{ml}$ final volume with dist. water. The mixture was kept at $50^{\circ} \mathrm{C}$ for $20 \mathrm{~min}$, followed by centrifugation at $2000 \mathrm{~g}$ for $10 \mathrm{~min}$. Afterwards, the supernatant was transferred to a cuvette and absorbance was read at $750 \mathrm{~nm}$. Results were presented as gallic acid equivalents (LiANHE et al., 2012).

\subsection{Oxidative stability analysis}

The oil sample (300 mg) was mixed with $9.9 \mathrm{ml}$ chloroform/methanol mixture $(7: 3, \mathrm{v} / \mathrm{v})$, then $50 \mu \mathrm{l}$ iron(II) chloride solution and $50 \mathrm{ml}$ xylenol orange $(10 \mathrm{mM})$ were added. The mixture was incubated at room temperature for $5 \mathrm{~min}$ and centrifuged at $1000 \mathrm{~g}$ for $5 \mathrm{~min}$. The absorbance was read at $560 \mathrm{~nm}$ with spectrophotometer (Li et al., 2014). Results were presented as milliequivalents $\mathrm{O}_{2} / \mathrm{kg}$ oil.

\subsection{Tocopherol contents}

A Waters 2695 Alliance high-performance liquid chromatography system equipped with a C18 column $(250 \times 4.6 \mathrm{~mm}$ i.d., $5 \mu \mathrm{m}$ particle size $)$ was used. Each sample was mixed with the mixture of methanol, hexane, and tetrahydrofuran (80:10:10), vortexed, and centrifuged to obtain the supernatant. The mixture of an equal volume of methanol and acetonitrile was used as mobile phase at the flow rate of $1 \mathrm{ml} \mathrm{min}^{-1}$. Fluorescence detector was used for the quantification of tocopherol contents at $290 \mathrm{~nm}$ excitation and $325 \mathrm{~nm}$ emission wavelengths (Constantin et al., 2013). 


\subsection{Statistical study}

For statistical analysis, a factorial design was implemented with two independent variables, storage days and level of antioxidant incorporation. All experiments were conducted in triplicate, and results were expressed by means of triplicate values with standard deviations. One-way analysis of variance (ANOVA) and Duncan's multiple range test were used for statistical analysis.

\section{Results and discussion}

\subsection{Rancimat analysis}

Table 1 revealed the significant effect $(\mathrm{P} \leq 0.05)$ of $\mathrm{PSO}$ on oxidative stability of plant-based oils. The lowest IP values of oil samples without PSO and BHA revealed that these samples oxidized easily during storage. Both oils showed significantly higher IP values with incorporated antioxidants as compared to blank oil samples. Canola oil showed the higher IP values as compared to sunflower oil possibly because of higher PUFA contents that play antioxidant role. Results indicate the strong antioxidant effect of PSO against oxidative deterioration due to its $\beta$-sitosterol, campesterol, stigmasterol, tocopherols, and PUFA contents as compared to synthetic antioxidants (SHABBIR et al., 2017). Similar results were observed by RAFIEE and co-workers (2012) while evaluating the protective effect of olive oil leaves extract in sunflower oil during storage.

Table 1. Induction period for canola oil and sunflower oil obtained by Rancimat analysis

\begin{tabular}{|c|c|c|c|c|}
\hline \multirow{2}{*}{ Oil samples } & \multicolumn{4}{|c|}{ Induction period (h) } \\
\hline & Control & BHA (200 ppm) & PSO $(5 \%)$ & PSO $(7 \%)$ \\
\hline Canola oil & $9.50 \pm 0.35^{\mathrm{d}}$ & $10.18 \pm 0.38^{\mathrm{c}}$ & $11.23 \pm 0.45^{\mathrm{b}}$ & $11.94 \pm 0.34^{\mathrm{a}}$ \\
\hline Sunflower oil & $1.85 \pm 0.04^{\mathrm{d}}$ & $2.28 \pm 0.09^{\mathrm{c}}$ & $3.26 \pm 0.10^{\mathrm{b}}$ & $3.87 \pm 0.13^{a}$ \\
\hline
\end{tabular}

Mean values in the same row followed by different superscript letters are significantly $(\mathrm{P}<0.05)$ different.

\subsection{Antioxidant potential of oils during storage}

Both DPPH and ABTS assays were conducted to assess the oxidative stability of plant-based oils during storage as shown in Figures 1 and 2 analysed by ANOVA (data of day 10, 20, 40, and 50 are not shown due to non-significant results). Initially, oils incorporated with PSO showed higher antioxidant capacities compared to positive and negative control oils. Canola oil showed higher antioxidant capacity compared to sunflower oil. However, a decreasing trend was observed in antioxidant capacity in all oil samples during storage. The maximum decrease was observed in blank oil samples compared to oils with PSO and BHA throughout storage. The higher oxidative stability of PSO incorporated oils is because of punicic acid, a conjugated $\alpha$-linolenic PUFA with potential antioxidant activity, body fat lowering, lipid metabolism normalizing, cytotoxic effects, and other health related benefits as reported by earlier studies (CARVAlHo Filho, 2014; ShabBir et al., 2017). 


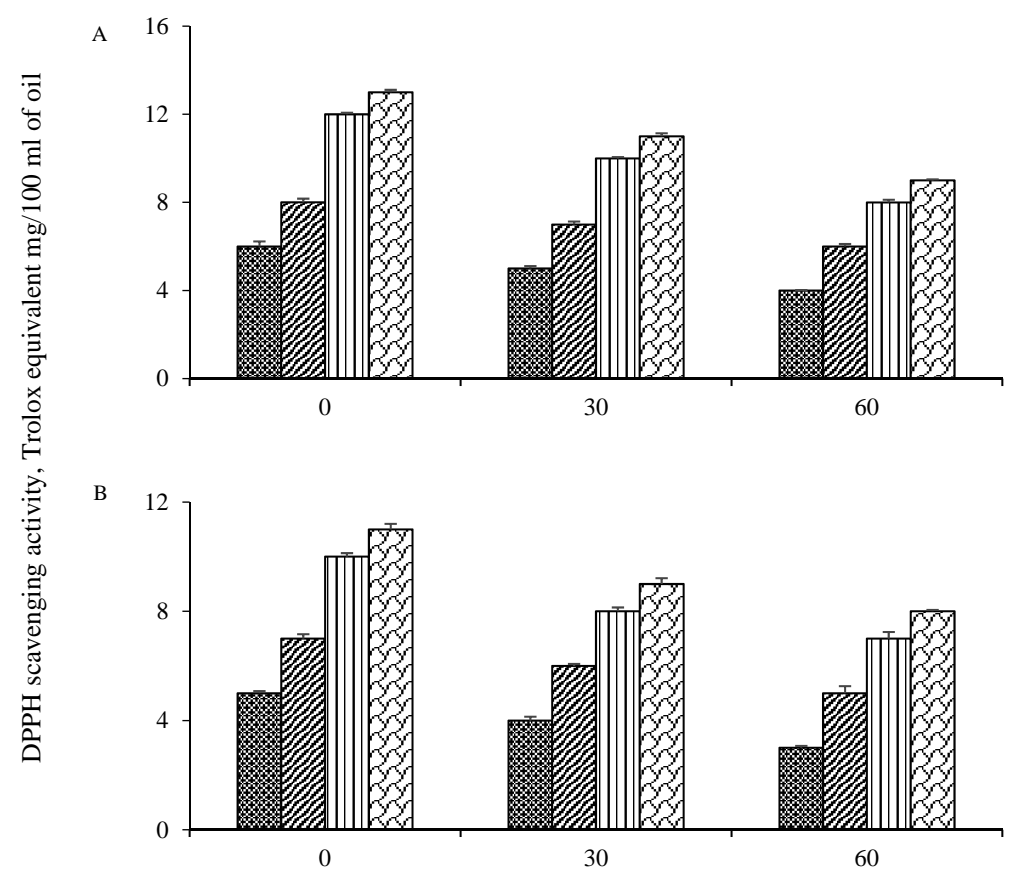

Fig. 1. Changes in antioxidant activity assessed by DPPH in oil blends of (A) canola oil (B) sunflower oil during storage

Fos: Control; $\mathbb{N}$ : BHA (200 ppm); 盂: PSO (5\%); 龱: PSO (7\%)

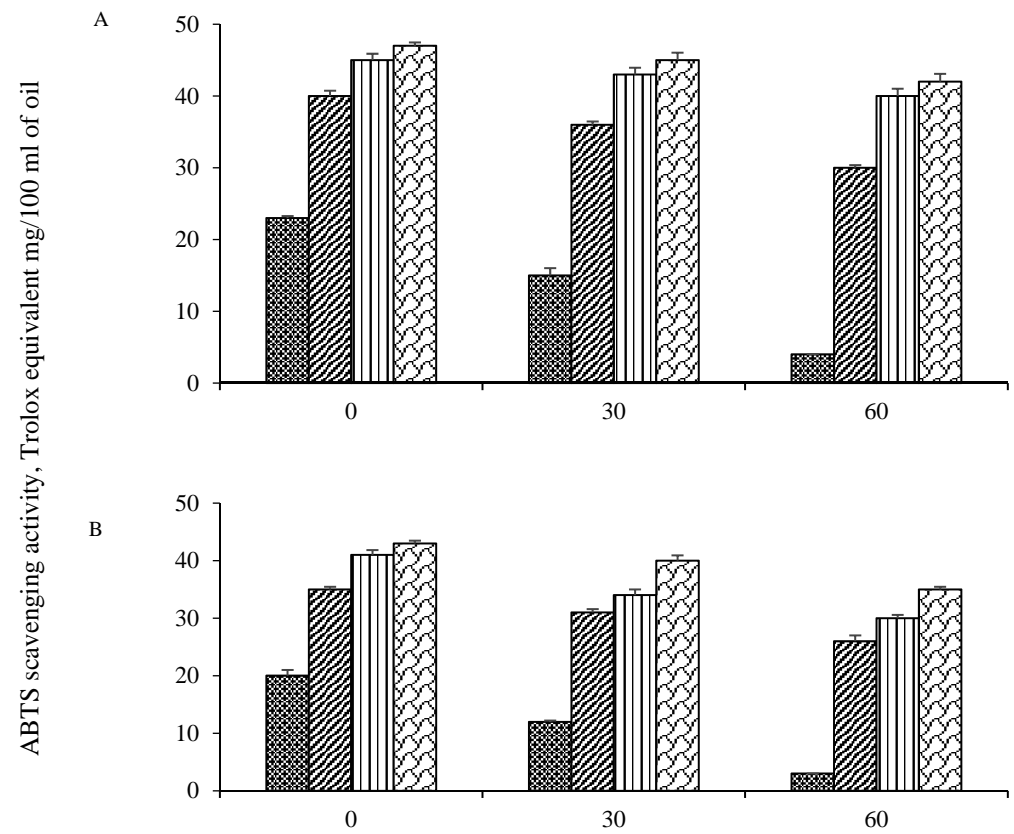

Fig. 2. Changes in antioxidant activity assessed by ABTS in oil blends of (A) canola oil (B) sunflower oil during storage

Fos: Control; $\mathbb{Q}$ : BHA (200 ppm); 岁: PSO (5\%); $\otimes:$ PSO (7\%) 
During storage, canola oil showed higher phenolic contents than sunflower oil samples as shown in Figure 3. As well, the highest phenolic contents were observed in oil samples with $7 \%$ PSO throughout storage. At day 0 , both canola and sunflower oils supplemented with 7\% PSO exhibited higher phenolic contents compared to blank and BHA oil blends, although a decreasing trend was observed for phenolic contents in all oil samples with or without PSO/BHA addition during storage. The maximum decrease was observed in blank oil samples of both canola and sunflower. PSO possesses bioactive compounds and phenolic contents, and decrease in phenolic contents during storage is maybe because of decomposition and oxidation of phenolics of oil bodies that ultimately undergo various changes in composition (YANG et al., 2016). Additionally, the phenolic compounds denote their $\mathrm{H}$ atom to free radicals to stabilize the oil system and provide protection against oxidation (SÁNCHEZ et al., 2007).

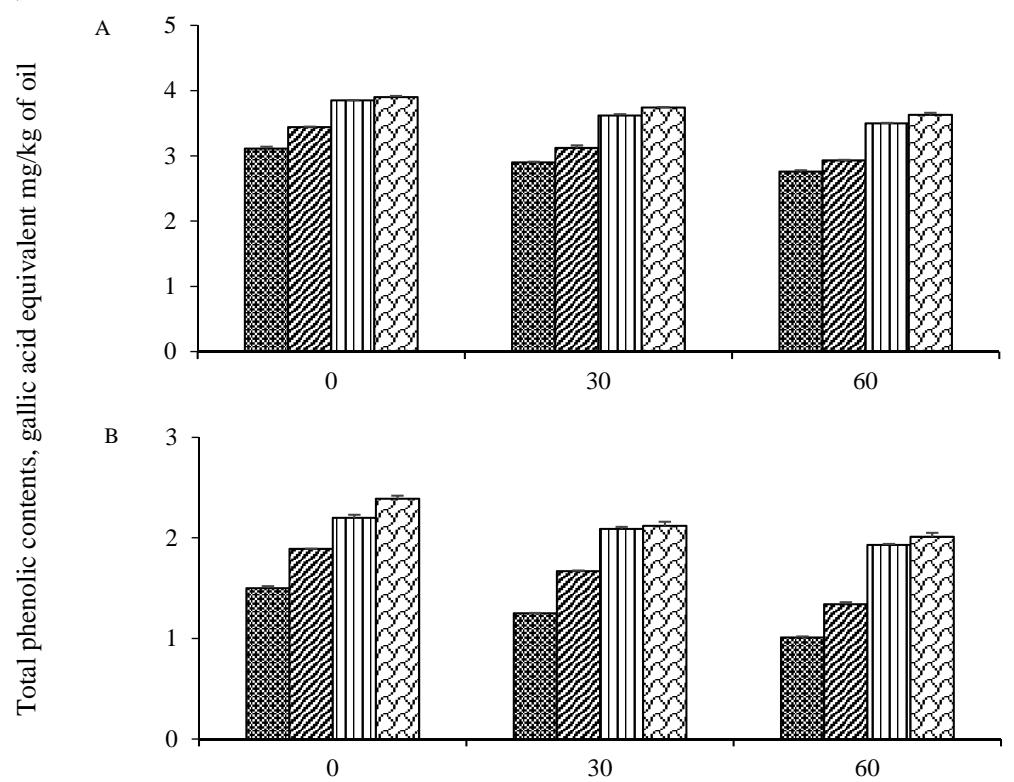

Fig. 3. Total phenolic contents in different blends of (A) canola oil (B) sunflower oil during storage Fo: Control; $\mathbb{\mathbb { N }}$ : BHA (200 ppm); 岨: PSO (5\%); : PSO (7\%)

\subsection{Oxidative stability analysis}

The changes that were observed in POV of oil samples during storage are shown in Figure 4. Data showed that POV of all oil blends increased sharply during storage. Initially, the POV of all oil samples were non-significant regardless of PSO/BHA addition. The maximum increase was observed in blank oil samples during storage in canola and sunflower oil samples, while the oil samples with PSO and BHA incorporation showed slight increase in POV, justifying the potential oxidation stability effect of PSO. The minimum increase was observed in oil samples with 7\% PSO incorporation. The PSO showed significant retarding effect against oil oxidation as compared to synthetic antioxidant BHA. The maximum oxidation retardation was observed in 7\% PSO oil samples in both plant-based oils. YANG and co-workers (2016) reported similar trend in POV in plant based oils (rice bran oil, cotton seed oil, and soybean oil) supplemented with rosemary extract as compared to synthetic 
antioxidants. Application of natural antioxidants with strong scavenging activity of bioactive compounds in food items is more feasible than synthetic ones (RAHMANIAN et al., 2015).
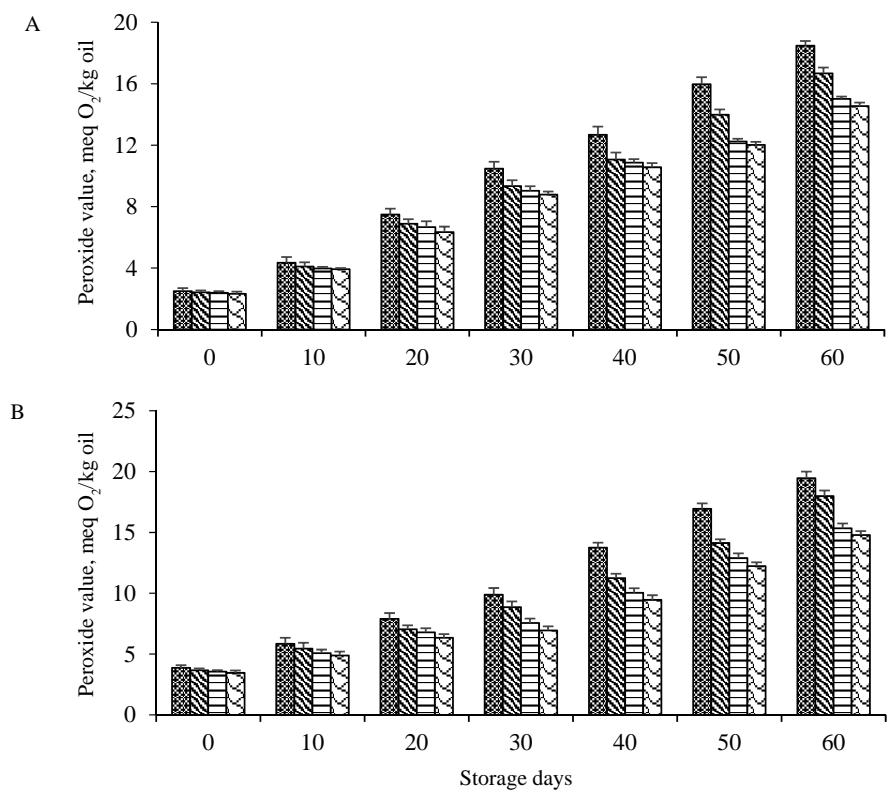

Fig. 4. Peroxide values (meq $\mathrm{O}_{2} / \mathrm{kg}$ oil) of different oil blends of (A) canola oil (B) sunflower oil during storage Fis: Control; $\mathbb{Q}:$ BHA (200 ppm); 盂: PSO (5\%); 园: PSO (7\%)

\subsection{Tocopherol contents}

Data in Table 2 show the significant effect $(\mathrm{P} \leq 0.05)$ of storage and PSO supplementation on tocopherol content in all oil blends. Both plant-based oils showed different amounts of individual and total tocopherol contents in each blend. Initially, sunflower oil possessed total tocopherol contents of $620.9 \mu \mathrm{g} \mathrm{g}^{-1}$ followed by canola oil containing $259.7 \mu \mathrm{g} \mathrm{g}^{-1}$ of total tocopherol in the blank oil sample. Major individual tocopherols found were $\boldsymbol{\alpha}-, \boldsymbol{\gamma}$-, and $\delta$-tocopherols in sunflower oil and $\alpha$ - and $\gamma$-tocopherols in canola oil samples. The amounts of $\alpha$-tocopherol were the highest in all samples of canola and sunflower oils throughout storage. Initially, highest $\alpha$-tocopherol contents were found in canola oil and sunflower oil with 7\% PSO incorporation. Similarly, the other tocopherol contents were also found in higher amount in 7\% PSO oil samples. However, a significant decreasing trend was observed in individual and total tocopherol contents in all oil samples during storage. The highest decreasing trend ( $38 \& 24 \%$ ) was observed for total tocopherols in blank oil samples of canola and sunflower oils. The lowest loss $(9.65 \%)$ was observed in 7\% PSO oil sample followed by $5 \%$ PSO (12\%) and BHA (15\%) oil samples for total tocopherol contents during storage. The data revealed that the addition of PSO slows down the degradation process of tocopherols more during storage than synthetic antioxidant. BEDDOws and co-workers (2001) observed the preservation effect of ascorbyl palmitate addition on tocopherol content in plant-based oils during storage resulting in delayed rancidity. Similarly, YANG and co-workers (2016) reported the same decreasing trend in tocopherol contents in plant based oil supplemented with rosemary extract during storage. 


\section{Conclusions}

Pomegranate seed oil was evaluated as an alternative antioxidant against oxidative deterioration of canola and sunflower oils during storage. Results showed that PSO is more effective in delaying oxidation in plant-based oils during storage when compared to synthetic antioxidants. The oil blends with 7\% PSO exhibited more stable configuration, i.e. higher IP value, better antioxidant capacity, lower POV, and slower degradation of tocopherols during storage. Consequently, the present findings suggest that PSO addition could be an efficient way to delay auto-oxidation in oils with improved nutritional attributes.

$*$

Authors want to pay gratitude to Higher Education Commission, Islamabad, Pakistan, for their financial support throughout the research study.

\section{References}

Abd-Elghany, M., Ammar, M. \& Hegazy, A. (2010): Use of olive cake extract as a natural antioxidant for improving the stability of heated sunflower oil. World Appl. Sci. J., 11, 106-113.

Antolovich, M., Prenzler, P.D., Patsalides, E., McDonald, S. \& Robards, K. (2002): Methods for testing antioxidant activity. Analyst, 127, 183-198.

Beddows, C.G., JAGAit, C. \& Kelly, M.J. (2001): Effect of ascorbyl palmitate on the preservation of $\alpha$-tocopherol in sunflower oil, alone and with herbs and spices. Food Chem., 73, 255-261.

Carvalho Filho, J.M. (2014): Pomegranate seed oil (Punica granatum L.): A source of punicic acid (conjugated $\alpha$-linolenic acid). J. Hum. Nutri. Food Sci., 2, 1-11.

Constantin, B., T Matea, C., Raducu, C., Miresan, V. \& Negrea, O. (2013): Tocopherol content in vegetable oils using a rapid HPLC fluorescence detection method. Not. Bot. Horti. Agrobo., 41, 93-96.

Cordeiro, A., Medeiros, M., Silva, M., Silva, I., Soledade, L., Souza, A., Queiroz, N. \& Souza, A. (2013): Rancimat and PDSC accelerated techniques for evaluation of oxidative stability of soybean oil with plant extract. $J$. Therm. Anal. Calorim., 114, 827-832.

Da Silva, A.C. \& Jorge, N. (2014): Bioactive compounds of the lipid fractions of agro-industrial waste. Food Res. Int., 66, 493-500.

JENNINGS, B.H. \& Акон, C.C. (2009): Effectiveness of natural versus synthetic antioxidants in a rice bran oil-based structured lipid. Food Chem., 114, 1456-1461.

LANSKy, E.P. \& NEWMAN, R.A. (2007): Punica granatum (pomegranate) and its potential for prevention and treatment of inflammation and cancer. J. Ethnopharmacol., 109, 177-206.

Li, Y., Li, D., Qi, B., Rokayya, S., Ma, W., Liang, J., Sui, X., Zhang, Y. \& Jiang, L. (2014): Heating quality and stability of aqueous enzymatic extraction of fatty acid-balanced oil in comparison with other blended oils. $J$. Chem. U. A. R., 2014, 8.

Lianhe, Z., XInG, H., Li, W. \& ZhengXing, C. (2012): Physicochemical properties, chemical composition and antioxidant activity of Dalbergia odorifera T. Chen seed oil. J. Am. Oil Chem. Soc., 89, 883-890.

Rafiet, Z., JAfari, S., Alami, M. \& Khomeiri, M. (2012): Antioxidant effect of microwave-assisted extracts of olive leaves on sunflower oil. J. Agr. Sci. Tech.- Iran, 14, 1497-1509.

RAHMANIAN, N., JAFARI, S.M. \& WANI, T.A. (2015): Bioactive profile, dehydration, extraction and application of the bioactive components of olive leaves. Trends Food Sci. Tech., 42, 150-172.

Ruge, C., Changzhong, R. \& Zaigui, L. (2012): The effects of different inactivation treatments on the storage properties and sensory quality of naked oat. Food Bioprocess. Tech., 5, 1853-1859.

Sánchez, C.S., González, A.T., García-Parrilla, M., Granados, J.Q., De La Serrana, H.L.G. \& Martínez, M.L. (2007): Different radical scavenging tests in virgin olive oil and their relation to the total phenol content. Anal. Chim. Acta, 593, 103-107.

Shabbir, M.A., Khan, M.R., Saeed, M., Pasha, I., Khalil, A.A. \& Siraj, N. (2017): Punicic acid: A striking health substance to combat metabolic syndromes in humans. Lipids Health Dis., 16, 99, 9 pages. 
Sreekumar, S., Sithul, H., Muraleedharan, P., Azeez, J.M. \& Sreeharshan, S. (2014): Pomegranate fruit as a rich source of biologically active compounds. Biomed. Res. Int., 2014, Article ID 686921, 12 pages.

Sui, X., Dong, X. \& Zhou, W. (2014): Combined effect of pH and high temperature on the stability and antioxidant capacity of two anthocyanins in aqueous solution. Food Chem., 163, 163-170.

Sun-Waterhouse, D., Zhou, J., Miskelly, G., Wibisono, R. \& Wadhwa, S. (2011): Stability of encapsulated olive oil in the presence of caffeic acid. Food Chem., 126, 1049-1056.

TAghvaei, M. \& JAFARI, S.M. (2015): Application and stability of natural antioxidants in edible oils in order to substitute synthetic additives. J. Food Sci. Tech., 52, 1272-1282.

Tehranifar, A., Selahvarzi, Y., Kharrazi, M. \& Bakhsh, V.J. (2011): High potential of agro-industrial by-products of pomegranate (Punica granatum L.) as the powerful antifungal and antioxidant substances. Ind. Crop. Prod., 34, 1523-1527.

Viuda-Martos, M., Fernández-López, J. \& Pérez-Álvarez, J. (2010): Pomegranate and its many functional components as related to human health: A review. Compr. Rev. Food Sci. F., 9, 635-654.

YANG, Y., Song, X., SuI, X., QI, B., WANG, Z., Li, Y. \& JiAng, L. (2016): Rosemary extract can be used as a synthetic antioxidant to improve vegetable oil oxidative stability. Ind. Crop. Prod., 80, 141-147. 